\title{
Structural Characterization of PPII Helical Oligoproline
}

\author{
Patrick Wilhelm, Bartosz Lewandowski, and Helma Wennemers* \\ ETH Zurich, Laboratory of Organic Chemistry, 8093, Zurich, Switzerland
}

\section{Introduction}

Detailed knowledge of the geometry and conformational dynamics of molecules used as molecular scaffolds is crucial for the design of functional compounds based on this architecture. Oligoproline is such a molecular scaffold that adopts in aqueous environments a left-handed helix, the Polyproline II (PPII)-helix [1]. Powder diffraction studies provided in the 1950s valuable low-resolution information about the PPII-helix [2]. They revealed basic parameters e.g., the all-trans configuration of the amide bonds and that every third residue is stacked on top of each other. However, in contrast to crystal structures of collagen and several other proteins with PPII-helical domains, crystallization of an oligoproline in a PPII-helical conformation had remained elusive. Our interest in the use of azidoproline-containing oligoprolines as functionalizable scaffolds [3-6] led us to revisit the crystallization of oligoprolines.

\section{Results and Discussion}

$N$-Terminal functionalization of a proline-hexamer with $p$-bromobenzoic acid provided $p$ - $\mathrm{Br}-\mathrm{C}_{6} \mathrm{H}_{4}-\mathrm{Pro}_{6}-\mathrm{OH}$ that crystallized by vapor diffusion from acetonitrile to tetrahydropyrane. This high-resolution structure provided detailed insight into the conformational properties of PPII-helical oligoprolines [7].

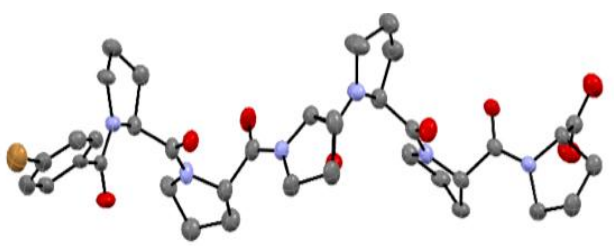

Fig. 2. Crystal structure of hexaproline $\mathrm{p}-\mathrm{Br}$ $\mathrm{C}_{6} \mathrm{H}_{4}-\mathrm{PrO}_{6}-\mathrm{OH}$ (ORTEP).

Distance measurements between every $\mathrm{N}, \mathrm{C} \alpha$, and $\mathrm{C}_{i}$ revealed that the helical pitch between every third residue is $8.98 \pm 0.14 \mathrm{~A}$ in contrast to the $9.36 \AA$ that was derived from the powder diffractogram [2]. Analysis of the distances between the carbonyl oxygens and the carbonyl carbons of neighboring residues $\left(\mathrm{O}_{i-I} \cdots \mathrm{C}_{i}\right)$ showed that they are all below the van der Waals' radii $(3.2 \AA)$ of these atoms (Figure 2). Such short distances are indicative of $\mathrm{n} \rightarrow \pi^{*}$ interactions between neighboring carbonyl groups, which involve delocalization of the non-bonding electrons of $\mathrm{O}_{i-l}$ into the $\pi^{*}$ orbital of the $\mathrm{C}_{i}=\mathrm{O}_{i}$ bond [8]. Additional parameters that are indicative for $\mathrm{n} \rightarrow \pi^{*}$ interactions are the degree of pyramidalization of $\mathrm{C}_{i}(\Delta)$ and the trajectory angle $\mathrm{O}_{i-1} \cdots \mathrm{C}_{i}=\mathrm{O}_{i}\left(\theta_{\mathrm{BD}}\right)$ between adjacent carbonyl groups. Ideal trajectory angles of $\sim 104^{\circ}$ were observed in Pro1 and Pro 2 that also show the highest degree of pyramidalization of $C_{i}(\Delta)$. These values of $0.040 \AA$ and $0.023 \AA$ are in fact the highest ever observed values for interactions between two amides. Additionally the crystal structure showed a correlation between the ring-pucker and the backbone dihedral angles and that water is not a necessity for PPII-helical oligoproline [7]. 
Next, we explored the dynamic behavior of oligoprolines and performed double electronelectron- resonance (DEER) measurements on a series of double spin-labelled oligoprolines to measure the dipole-dipole interactions between spin labels at distances on the nanometer scale with very high precision [8]. In contrast to commonly applied FRET chromophores, these spin-labels are significantly smaller and therefore reduce label-related line-broadening on the distance distribution. We used the super high-spin complex $\mathrm{Gd}^{3+}$-DOTA and

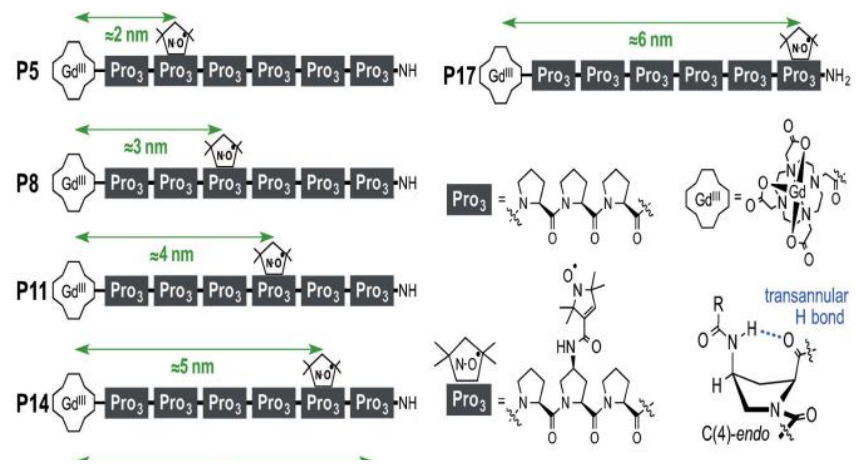

Fig. 3 a.) Double spin-labeled oligoprolines P5, P8, P11, P14, and P17 b.) C(4)-endo pucker of (4S)Amp.

the nitroxide radical 2,2,5,5-tetramethyl-3-pyrrolin-1-oxyl as spin labels and varied the distances across a series of five oligoproline of the same length (Figure 3). Narrow distance distributions were obtained from the DEER-measurements both, in trifluoroethanol (TFE) and a water/glycerol mixture with maxima at distances that were expected for PPII-helices. The narrowest distance distributions were observed for probe P5, with distance distribution widths increasing with the number of repeating $\mathrm{PrO}_{3}$ units between the labels in the other probes P8-P17. This implies that the width and shape of the distributions of P11, P14, and P17 are governed by the flexibility of the oligoproline backbone. This observation is typical for shape-persistent oligomers, which is predicted by the worm-like-chain model (WLC) and related to the persistence length. [10]

Thus, using the WLC model the persistence length at ambient temperature $(298 \mathrm{~K})$ was determined to be between 3.0 and $3.5 \mathrm{~nm}$.

Monte Carlo Simulations allowed to separate influences of backbone flexibility and the occurrence of cis-amide bonds that contribute to the observed line-broadening [11]. These computational studies allowed to quantify the probability of a cis-amide bond to be $\sim 2 \%$ in both solvents [12].

In conclusion, we have gained insight into the crucial parameters of oligoprolines from the first crystal-structure of a PPII-helical oligoproline [7] and determined their shape-persistence [12]. The presented data are crucial for the use of oligoprolines as molecular scaffolds and will allow for the design of even better tailored molecular systems [7,12].

\section{References}

1. M. De Zotti, M., et al. J. Pept. Sci. 20, 307-322 (2014), http://dx.doi.org/10.1002/psc.2638

2. Cowan, P.M., et al. Nature, 176, 501-503 (1955), http://dx.doi.org/10.1038/176501a0

3. Kumin, M., et al. J. Am. Chem. Soc. 129, 466-467 (2007), http://dx.doi.org/10.1021/ja067148o

4. Upert, G., et al. Angew. Chem. Int. Ed. 51, 4231-4234 (2012), http://dx.doi.org/10.1002/anie.201107183

5. Lewandowska, U., et al. Angew. Chem. Int. Ed. 53, 12537-12541 (2014), http://dx.doi.org/10.1002/anie.201408279

6. Kroll, C., et al., J. Am. Chem. Soc. 135, 16793-16796 (2013), http://dx.doi.org/10.1021/ja4087648

7. Wilhelm, P., et al. J. Am. Chem. Soc. 136, 15829-15832 (2014), http://dx.doi.org/10.1021/ja507405j

8. Hinderaker, M.P., et al. Protein Sci. 12, 1188-1194 (2003), http://dx.doi.org/10.1110/ps.0241903

9. Pannier, M., et al. J. Magn. Reson. 142, 331-340 (2000), http://dx.doi.org/10.1006/jmre.1999.1944

10.Jeschke, G., et al. J. Am. Chem. Soc. 132, 10107-10117 (2010), http://dx.doi.org/10.1021/ja102983b

11.Best, R.B., et al. Proc. Natl. Acad. Sci. USA 104, 18964-18969 (2007),

http://dx.doi.org/10.1073/pnas.0709567104

12.Garbuio, L., et al. Chemistry - Eur.J. 21, 10747-10753 (2015), http://dx.doi.org/10.1002/chem.201501190 